\title{
Multifeature Extraction of Three-Dimensional Topography of Carbon Steel Specimen during Fatigue Process
}

\author{
Tao Liu $\left(\mathbb{D},{ }^{1,2}\right.$ Jingxiong Wu $\left(\mathbb{D},{ }^{1}\right.$ Jingfa Lei $\mathbb{D}^{1,2}$ Xue Wang $\mathbb{D}^{1},{ }^{1}$ Bingqi Zhang $\mathbb{D}^{1}{ }^{1}$ \\ and Shu Zhang $\mathbb{D}^{1,2}$ \\ ${ }^{1}$ School of Mechanical and Electrical Engineering, Anhui Jianzhu University, Hefei, Anhui 230601, China \\ ${ }^{2}$ Anhui Education Department Key Laboratory of Intelligent Manufacturing of Construction Machinery, Hefei, \\ Anhui 230601, China \\ Correspondence should be addressed to Tao Liu; liutao19841015@163.com
}

Received 13 October 2020; Revised 4 January 2021; Accepted 13 January 2021; Published 27 January 2021

Academic Editor: Jun Liu

Copyright (C) 2021 Tao Liu et al. This is an open access article distributed under the Creative Commons Attribution License, which permits unrestricted use, distribution, and reproduction in any medium, provided the original work is properly cited.

In order to investigate the variation of three-dimensional metal surface topography during fatigue process, a three-dimensional (3D) topography acquisition platform was built with an in situ tensile tester and a three-dimensional profilometer. Q235 steel specimens were chosen as research objects, and the three-dimensional surface topography information at various stages of fatigue damage was obtained. Through the characterization of three-dimensional roughness, combined with surface height distribution and multifractal analysis, the variations of metal surface topography in the fatigue process were described. Results show that the arithmetic mean deviation of the surface $\left(S_{a}\right)$, the width of the multifractal spectrum $(\Delta \alpha)$, and the mean value of surface height distribution $(\mu)$ and its standard deviation $(\delta)$ increase nonlinearly with the increase of fatigue cycles. The rate of fatigue damage is slow in the early stage and high in the middle and late stages. The surface height distribution amplitude (A) decreases with the increase of fatigue cycles, which indicates that the height data concentration decreases, and the metal surface becomes uneven. The Bayesian data fusion method was applied to establish a nonlinear mapping between the topography features and the damage, with the above five characteristic parameters $\left(S_{a}, \Delta \alpha, \mathrm{A}, \mu\right.$, and $\left.\delta\right)$ as the data layer. Finally, a surface topography feature fusion method is proposed, and a case study is conducted to verify its applicability. The research results can provide reference for fatigue damage assessment.

\section{Introduction}

Metal surface topography is closely related to its fatigue strength and wear resistance, which affects the service life and reliability of components. When metal components, especially polycrystalline material components, are subjected to cyclic loading, the slip generated by the persistent slip band in the grains of the surface can be largely transferred, which makes the surface rough and causes corresponding changes in topographical characteristics [1]. The variation of surface topography is applied to describe fatigue damage, which is expected to bring new direction and ideas for fatigue damage assessment.

At present, several methods have been proposed to describe the change of metal surface topography, such as two-dimensional (2D) gray-scale image processing of metal surfaces and feature extraction of energy and entropy parameters from the gray level co-occurrence matrix [2-4]. In addition, the laser speckle pattern is also applied to characterize the surface roughness [5]. In the research of surface topography evolution during the fatigue damage process, $2 \mathrm{D}$ and $3 \mathrm{D}$ roughness parameters are adopted to explore the inherent relationship between damage degree and topography [6-9]. It is found that, with the increase of fatigue damage degree, the roughness, frequency distribution, and fractal dimension of the surface profile are also increased [10]. The linear correlations between fatigue damage accumulation and roughness and between roughness and stiffness degradation are elucidated [11]. A composite damage index, which can reflect the accumulation of surface 
topography and topological changes in each fatigue damage state [12], is proposed, and a crack path prediction algorithm is established on this basis [9]. However, in the process of $3 \mathrm{D}$ surface topography extraction, even if a high-precision image acquisition instrument is utilized, the gray-scale image obtained often suffers from poor accuracy after 2D gray-scale processing, and the characteristic data is susceptible to environmental interference. The roughness parameters that characterize the topography, such as $R a$ (the arithmetic mean deviation of the profile), are scale-dependent quantities, and it is arduous to describe the self-similarity, hierarchical characteristics, and detailed changes of surface topography.

Surface height information and its distribution provide the basis for extracting surface topography features. Meanwhile, fractal description has the advantage of scale invariance, which does not depend on the observation scale and resolution of the instrument, and can reflect the selfsimilarity of the rough surface structure at different scales $[13,14]$. In this paper, Q235 steel specimens are selected to perform the axial-tensile fatigue test. The surface height information under various fatigue cycles is collected to obtain 3D roughness parameters such as the surface arithmetic mean deviation. The amplitude, mean value, and standard deviation characteristics of surface height distribution in the fatigue process are analyzed, and the multifractal spectrum characteristics from the early stage of fatigue to fracture are obtained. Based on the Bayesian data fusion method, a multifeature data fusion evaluation model of surface topography in the fatigue damage process is established.

\section{Multifeature Analysis of 3D Surface Topography during the Fatigue Damage Process}

2.1. Basic Characteristic of 3D Surface Topography. In the field of mechanical engineering, 3D surface topography refers to the microscopic characteristics of the surface of parts, such as peak-valley characteristics, texture direction, and shape deviation. These characteristics are often directly related to the surface properties of parts (fatigue strength, wear resistance, corrosion resistance, etc.). When the metal material/component is subjected to cyclic loading, the cyclic slip (extrusion and intrusion) leads to crack nucleation, and the irreversibility of shear displacement along the slip band makes the material surface rough. The rough surface is shown in the form of micropeak (extrusion) and valley (intrusion).

The traditional measurement and evaluation of surface topography is mainly based on $2 \mathrm{D}$ and $3 \mathrm{D}$ surface roughness. The $3 \mathrm{D}$ roughness can reflect the microtopography and performance characteristics of the target surface more comprehensively and intuitively. ISO 25178 divides 3D surface roughness parameters into six types: height parameters, spacing parameters, function and related parameters, mixing parameters, characteristic parameters, and other parameters $[15,16]$. The most commonly used is the arithmetic mean deviation $S_{a}$ of height parameters. The schematic diagram of the surface arithmetic mean deviation $S_{a}$ (unit: $\mu \mathrm{m}$ ) is shown in Figure 1, which refers to the arithmetic average height in the sampling area.

The mathematical expression is as follows:

$$
S a=\frac{1}{A} \iint_{A}|z(x, y)| \mathrm{d} x \mathrm{~d} y,
$$

where $A$ is the sampling area and $z(x, y)$ is the height of the point at $(x, y)$.

\subsection{Multifractal Description of Metal Surface Topography} during the Fatigue Process. Multifractal, also known as fractal measure, is a singular combination of multiple subsets, and each subset has different fractal behavior. The fractal dimension distribution of multifractal is uneven, which is based on the extension of the original fractal concept to the nonuniform fractal, and its description of the fine structure of the fractal is hierarchical. The surface topography of the metal fatigue process is highly nonlinear and complex, and the use of multifractal can describe its local characteristics more effectively. The calculation process of multifractal parameters is as follows.

The surface height matrix of the metal fatigue process is divided into $n$ regions $S_{i}(i=1,2,3, \ldots, n)$. Let $\varepsilon_{i}$ be the scale size of the $i$ th region and $P_{i}$ be the generating probability of the region $S_{i}$. For each region with scale size $\varepsilon$, the generating probability is as follows:

$$
P_{i}(\varepsilon)=\frac{S_{i}(\varepsilon)}{\sum S_{i}(\varepsilon)},
$$

where $S_{i}(\varepsilon)$ represents the sum of the height values at all positions of the $i$ th area and $\sum S_{i}(\varepsilon)$ indicates the sum of heights at the whole area.

In a scale-free self-similar area, the following relationship exists for probability $P_{i}(\varepsilon)$ :

$$
P_{i} \propto \varepsilon_{i}^{\alpha_{i}},
$$

where $\alpha$ is the singularity scale index, which is a quantity reflecting the degree of singularity of each small area in fractal.

The relationship between the number of probability subsets $N_{\alpha}(\varepsilon)$ (corresponding to $\alpha$ ) and $\varepsilon$ under the scale $\varepsilon$ is defined as the following:

$$
N_{\alpha}(\varepsilon) \propto \varepsilon^{-f(\alpha)},
$$

where $N_{\alpha}(\varepsilon)$ is the number of probability subsets corresponding to $\alpha$ under the scale $\varepsilon$ and $f(\alpha)$ is the fractal dimension with the same $\alpha$ subset, and the multifractal spectrum is composed of $\alpha$ and $f(\alpha)$ under different scales.

Let the partition function $\chi_{q}(\varepsilon)$ be the weighted sum of the probability $P_{i}(\varepsilon)$ to the power of $q$ :

$$
\chi_{q}(\varepsilon)=\sum P^{q}(\varepsilon),
$$

where $q$ is the weighting factor. 


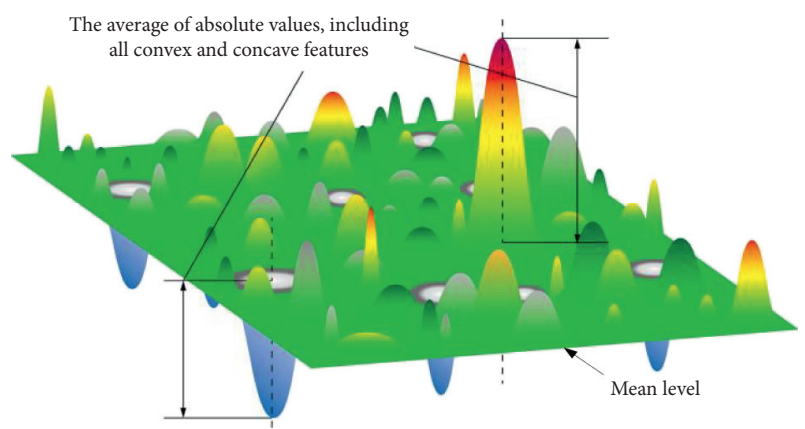

Figure 1: Schematic diagram of the surface arithmetic mean deviation $S_{a}$

Using the unified parameter measurement $u(q, \varepsilon)$ for calculation,

$$
u(q, \varepsilon)=\frac{P^{q}}{\sum P^{q}(\varepsilon)} .
$$

If $q>1$, the high probability set plays the main role, and if $q<1$, the low probability set plays the main role. The weighting function is to have a more comprehensive understanding of the fine structure on the target surface.

The calculation formula of the multifractal spectrum is as follows:

$$
\left\{\begin{array}{l}
\alpha(q)=\lim _{\varepsilon \longrightarrow 0} \frac{\sum u(q, \varepsilon) \ln P(\varepsilon)}{\ln (\varepsilon)}, \\
f(q)=\lim _{\varepsilon \longrightarrow 0} \frac{\sum u(q, \varepsilon) \ln u(q, \varepsilon)}{\ln (\varepsilon)} .
\end{array}\right.
$$

The multifractal spectrum image can be obtained by using all the $\alpha$ and $f(\alpha)$ data. The common characteristic parameter of the multifractal spectrum $\alpha-f(\alpha)$ is multifractal spectral width $\Delta \alpha=\alpha_{\max }-\alpha_{\min } . \Delta \alpha$ characterizes the uneven distribution of objects with fractal characteristics, and it can reflect the fluctuation of the elevation signal. The larger the $\Delta \alpha$ value, the more severe the elevation fluctuation, and the smaller the $\Delta \alpha$ value, the smaller the elevation fluctuation.

\subsection{Metal Surface Height Distribution and Numerical} Characteristics during Fatigue. The random surface topography is determined by the height distribution with position coordinates as variables. It is difficult to describe surface topography using strict mathematical expressions. However, the statistical parameters of surface topography can be used for the evaluation of surface topography. Statistical frequency distribution refers to the interval division of each observation value according to a certain interval length so that each observation value forms a different interval distribution. Due to the difference in the nature of objective phenomena, their frequency distribution curves will show different shapes. The height distribution is a reflection of the surface profile shape, which plays an important role in the study of the size, shape, and distribution of the surface profile. Therefore, the surface height distribution can be adopted to statistically describe the changes in metal surface topography during fatigue.

Normal distribution, also known as Gaussian distribution, is the most common probability distribution of continuous random variables. The common physical quantity that obeys normal distribution includes the measurement error of object size, the measurement of biological shape, and the quality index of products under normal production conditions. On the basis of normal distribution, combined with the specific parameter of surface height data, the statistical analysis of surface height distribution in the metal fatigue process is carried out. The details are as follows.

If the distribution of surface height $(z)$ of the fatigue process satisfies

$$
f(z)=\frac{1}{\sqrt{2 \pi} \sigma} e^{-(z-\mu)^{2} / 2 \sigma^{2}}, \quad-\infty<z<+\infty,
$$

then the metal surface height in the fatigue process obeys the normal distribution, which is recorded as follows:

$$
z \sim N\left(\mu, \sigma^{2}\right) .
$$

In equation (9), $\mu$ is the mathematical expectation (mean value) and the position parameter, which determines the location of the distribution, that is, the dominant numerical value of the surface height in the process of metal fatigue. $\sigma$ (the standard deviation) is the scale parameter. When $\mu$ is a certain value, the shape of the curve is determined by $\sigma$, and the smaller the $\sigma$ value is, the higher and thinner the curve is, which indicates that the metal surface height distribution is more concentrated, the uniformity is better, and the surface is smoother. The larger the $\sigma$ value is, the more squat the curve is, which indicates that the metal surface height distribution is more dispersed, the uniformity is worse, and the surface is more rough and uneven. In addition, the amplitude A of surface height distribution is the frequency corresponding to the highest point of the normal distribution curve of surface height.

2.4. Surface Topography Feature Fusion Method. Naive Bayesian classifier is a simple and effective linear classifier. When the features are independent of each other, especially in the case of small-scale samples, the Bayesian model can show a good classification effect because of the small amount of sample data, and the feature variables are independent of each other, so the Bayesian method is applied for surface feature fusion.

There are three kinds of Bayesian models: the polynomial model, Gaussian model, and Bernoulli model. The polynomial model and Bernoulli model are suitable for discrete data variables, while the Gaussian model is suitable for continuous data variables. In this paper, the Gaussian model is used as the fusion classification model of sample surface features.

The Naive Bayesian method is a learning method using the Bayesian formula. The Bayesian method forms prior distribution by quantifying prior information and applies 
prior distribution to statistical inference to improve the accuracy of statistical inference. Naive means that it assumes the components of input variables (vectors) are independent of each other:

$$
P\left(\frac{X}{Y}\right)=\frac{P(X / Y)^{*} P(Y)}{P(X)}
$$

where $P(Y)$ is the prior probability, $P(X / Y)$ is conditional probability, obtained through historical data statistics, and $P(Y / X)$ is the posterior probability, which is the target.

The Gaussian model is a kind of the Naive Bayesian model. It assumes that each feature $x_{i}$ obeys normal distribution under each category $y$. The probability density function of normal distribution is adopted to calculate the probability in the algorithm as follows:

$$
P\left(\frac{x_{i}}{c_{i}}\right)=\frac{1}{\sqrt{2 \pi \sigma^{2}}} \exp \left(-\frac{\left(x_{i}-\mu\right)^{2}}{2 \sigma^{2}}\right),
$$

where $\mu$ represents the mean value of feature $x_{i}$ in the sample of category $c_{i}, i=1,2,3, \ldots$ :

$$
\mu=\frac{\sum_{i}^{n} x_{i}}{N}
$$

$\sigma^{2}$ stands for the variance of feature $x_{i}$ in the sample of category $y$ :

$$
\sigma^{2}=\frac{\sum_{i}^{n}\left(x_{i}-\mu\right)^{2}}{N}
$$

where $N$ is the number of training samples.

It is known from the Naive Bayesian formula that, for the posterior probability values of different categories $c_{i}$, the denominator of the Bayesian formula is always the same, so only the numerator is required to be solved. Taking category $c_{1}$ as an example, the posterior probability formula is as follows:

$$
P\left(c_{1} \mid x_{i}\right)=P\left(c_{1}\right) \cdot \prod_{i=1}^{n} P\left(x_{i} / c_{1}\right) \quad(\mathrm{i}=1,2,3 \ldots) .
$$

In the same way, the posterior probabilities of other categories can be obtained.

As described in Sections 2.1-2.3, the five features describing the surface topography are arithmetic mean deviation $S_{a}$, surface height distribution amplitude $\mathrm{A}$, mean value $\mu$, standard deviation $\sigma$, and multifractal spectral width $\Delta \alpha$, respectively. The above features correspond to $x_{i}$ in equation (11). The features are independent of each other and meet the requirements of the Naive Bayesian method. The feature data are all continuous variables, so the Gaussian model is selected as the fusion model. In the Gaussian model, the mean and variance of fatigue damage states (i.e., cyclic loading cycles, corresponding to $c_{i}$ of equation (11)) are calculated, and then, the probability density function of normal distribution is obtained. According to the probability density function, the probability density value of continuous test data is calculated to obtain the prior probability. The posterior probability value corresponding to each fatigue damage state is obtained by substituting the probability density value into the posterior probability formula equation (14). The fatigue damage state corresponding to the maximum posterior probability value is the prediction result.

\section{An Application Case Study}

3.1. Experimental Materials. Q235 steel specimens (GB/T 3075-2008) are selected as the experimental objects. The specimens are obtained by laser cutting of the same batch of materials. The geometric size of the specimen (unit: $\mathrm{mm}$ ) is shown in Figure 2.

The material composition is as follows: C:0.22\%, Mn: 0.162\%, Co:0.10\%, Cr:0.01\%, Zn:0.015\%, Pb:0.062\%, Mo: 0.003\%, P:0.112\%, S:0.101\%, Si:0.411\%, K:0.518\%, and Fe: Bal. The tensile strength is $383 \mathrm{MPa}$.

3.2. Experimental Process. The experimental platform (Figure 3) consists of an in situ tensile tester (Mtest5000-FK, Sinotest Equipment Co., Ltd.) and a three-dimensional profilometer (VR3200, Keyence). The in situ tensile tester is loaded using a piezoelectric stacking device with a deformation measurement accuracy of $1 \mu \mathrm{m}$, displacement resolution of $0.01 \mu \mathrm{m}$, and test force error of $\pm 1 \%$ of the indicated value. The $3 \mathrm{D}$ profilometer is based on the principle of grating projection for three-dimensional measurement, with a measurement accuracy of $3 \mu \mathrm{m}$ in height, $2 \mu \mathrm{m}$ in width, and a repeatability of $0.5 \mu \mathrm{m}$ in both height and width.

Four specimens were selected for the experiment, numbered 1, 2, 3, and 4. The fiber laser marking machine (JD-GQ20) was used for treating the surface of specimens in the raster scanned format to keep the surface state consistent. The processing area is shown in Figure 4.

Low-cycle fatigue tests were carried out on four specimens by the in situ tensile tester. The loading conditions are as follows: constant frequency $13 \mathrm{~Hz}$, loading average value $740 \mathrm{~N}$, and amplitude $60 \mathrm{~N}$. In the process of the fatigue test, under the same conditions, the surface topographic characteristics of four specimens were obtained after 0, 2000, 4000,6000 , and 8000 cycles and fracture, respectively. It is found that all the specimens are fractured within the range of $9700 \pm 200$ cycles.

In order to distinguish from surface waviness and surface shape error when collecting 3D surface topography, the standard Gaussian filtering method is used to remove components with the wave distance greater than $1 \mathrm{~mm}$. When extracting surface topography data, data points are taken from the center of the specimen outward to form a $128 * 128$ data region. The 


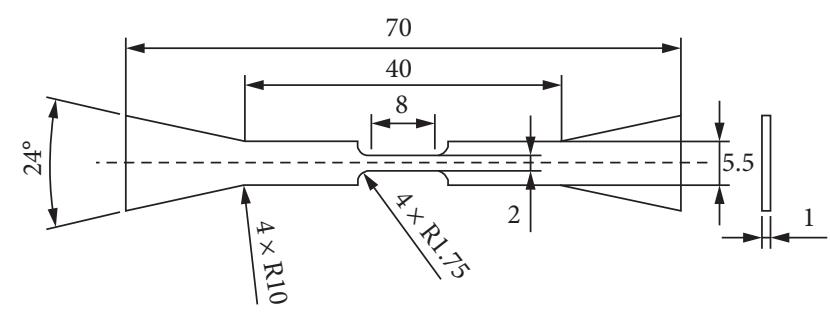

FIgURe 2: Specimen size (unit: $\mathrm{mm}$ ).

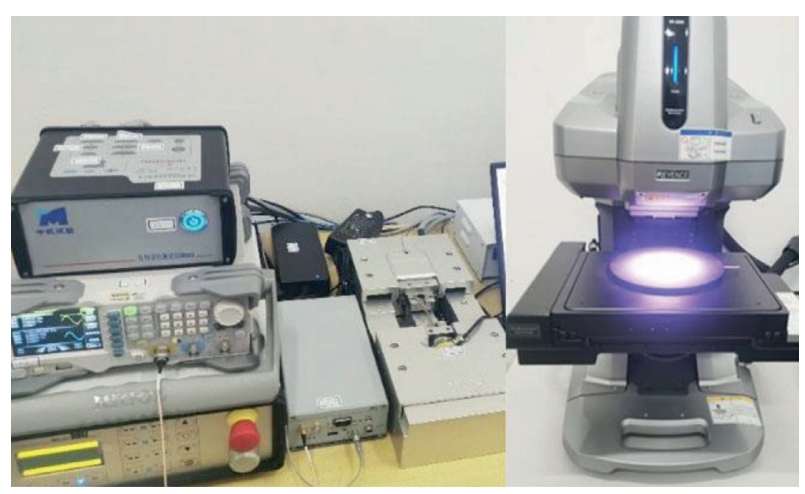

FIGURE 3: The experimental platform.

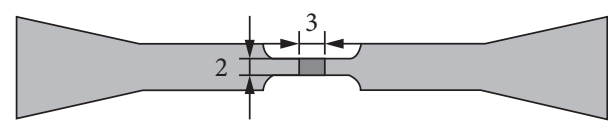

Figure 4: Dimensions of the processing area of the specimen (unit: $\mathrm{mm}$ ).

distance between any two data points is $7.39 \mu \mathrm{m}$, and the region size is $0.939 \mathrm{~mm} \times 0.939 \mathrm{~mm}$.

\section{Result Analysis}

4.1. 3D Surface Topography Evolution and Its Analysis. In order to visually observe the changes of three-dimensional surface topography of specimens subjected to cyclic loading, the three-dimensional surface topography maps of specimens under different fatigue cycles are drawn (taking no. 1 specimen as an example, the number of cycles is 0,2000 , $4000,6000,8000$, and fracture), as shown in Figure 5. It can be seen that the surface of the specimen without cyclic loading is relatively uniform, with fewer prominent peaks and valleys. With the increase of the number of cycles, the density of the surface peaks and valleys gradually increase, and the peak and valley values also increase correspondingly, showing obvious intrusion and extrusion effects.

After the fatigue fracture of the specimen, macro- and microanalyses of the fracture were performed. Figure 6(a) shows the macroscopic morphology of the fatigue fracture, which is composed of the fatigue crack initiation region, stable fatigue crack propagation region, and final fracture region. The crack originates from the position of stress concentration on the surface of the specimen and propagates uniformly to the inside. Due to repeated extrusion and friction, the fatigue crack initiation region is brighter, and the fracture surface of the final fracture region is dark gray and rough fibrous.

Scanning electron microscopy (Zeiss EVO18) was used to observe the fatigue fracture of the specimen, and SEM images of the fracture were obtained, as shown in Figures 6(b)-6(d).

Figure 6(b) shows the micromorphology of the fatigue crack propagation region, in which there are obvious wavy fatigue striations extending from the initiation region to the interior of the specimen. Figure 6(c) shows the micromorphology of the final fracture region. With the application of cyclic loading, the microcracks continue to expand and connect to form macrocracks, and the fracture morphology is similar to that of static load fracture. There are a large number of dimples in the fracture of the final fracture region, which is ductile fracture. When the fracture is magnified 2000 times, as shown in Figure 6(d), there are second phase particles at the bottom of dimples. The toughness of the second phase particles is poor, which will cause higher stress concentration during crack propagation and may lead to the appearance of secondary cracks.

4.2. Change in the Basic Characteristics of 3D Surface Topography during Fatigue. At the initial stage of the metal subjected to cyclic loading, dislocations are arranged in the material matrix as dislocation walls. With the increase of fatigue cycles, the dislocation arrangement becomes orderly; meanwhile, the thin straight and evenly distributed slip lines appear on the crystal grain surface. When the cyclic loading continues to act, the slip line widens and forms slip bands. With the widening of the slip band, the metal surface roughness changes due to the intrusion and extrusion phenomenon.

Figure 7 shows the variation curve of the surface arithmetic mean deviation $S_{a}$ during the fatigue process. $S_{a}$ presents a nonlinear growth trend with the increase of fatigue cycles.

4.3. Multifractal Spectrum Analysis. Figure 8 presents the multifractal spectrum of four specimens from the initial state to fatigue fracture. The value range of the weight factor $q$ is $[-55,55]$, and the interval is 1 . It can be seen from the figure that all the spectra are convex unimodal functions, indicating that the surface topography of the specimens exhibits various fractal characteristics under different cycles, that is, the metal surface topography has the multifractal characteristic during the fatigue process. Therefore, the multifractal spectrum can be applied to describe the change of metal surface topography.

Multifractal spectrum width $\Delta \alpha$ is a characteristic parameter of the multifractal spectrum, $\alpha_{\max }$ is the maximum value of the singularity index, and $\alpha_{\min }$ is the minimum value of the singularity index, which corresponds to the minimum and maximum height probabilities, respectively. $\Delta \alpha$ reflects the surface height fluctuation of the metal fatigue 

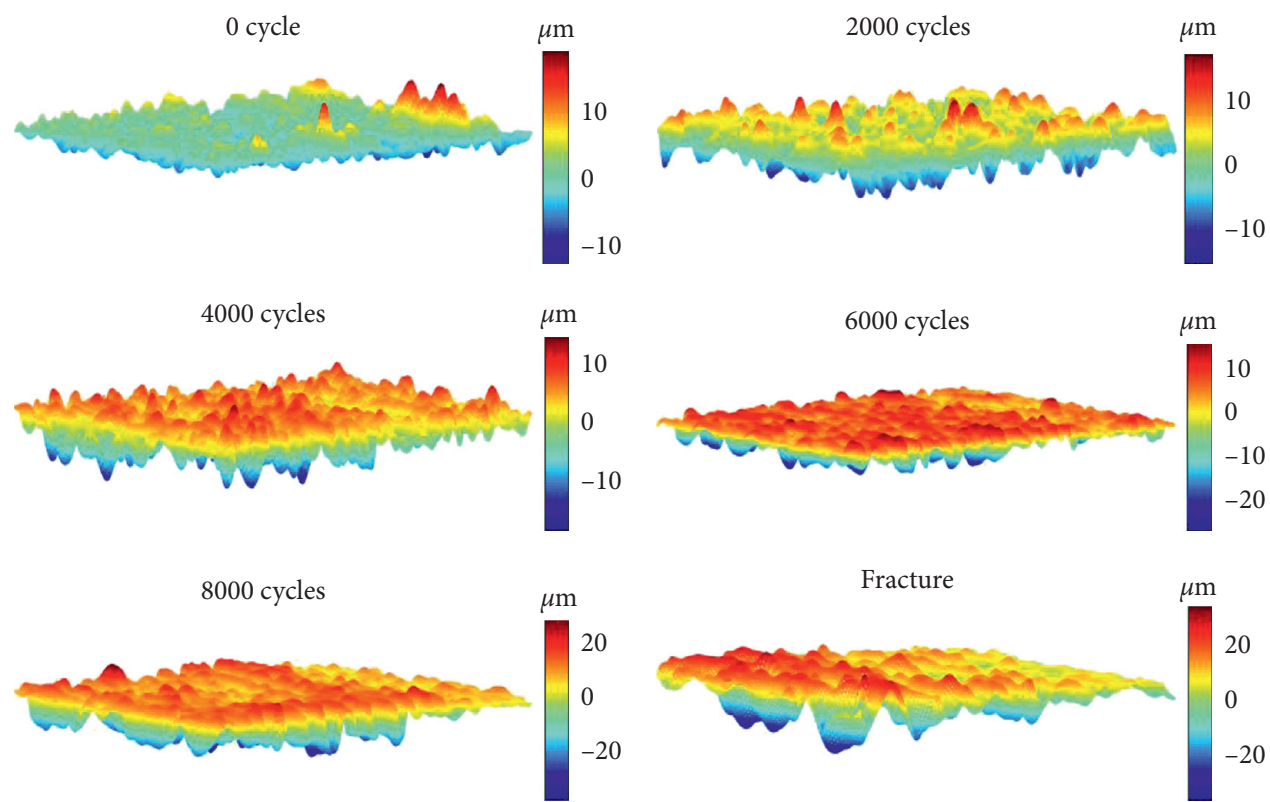

FIgURE 5: 3D surface topography of the specimen during fatigue.

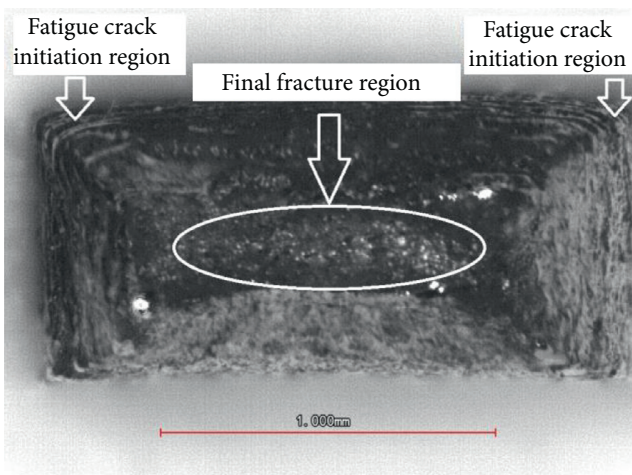

(a)

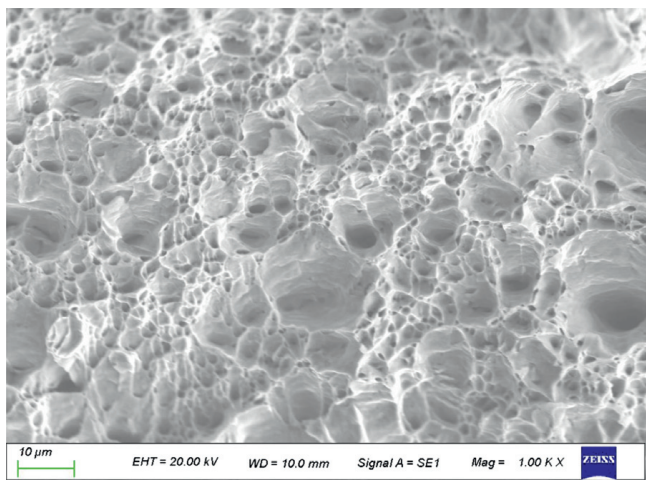

(c)

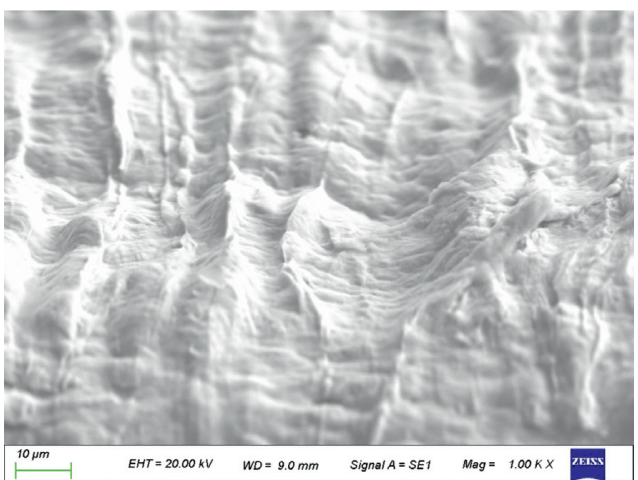

(b)

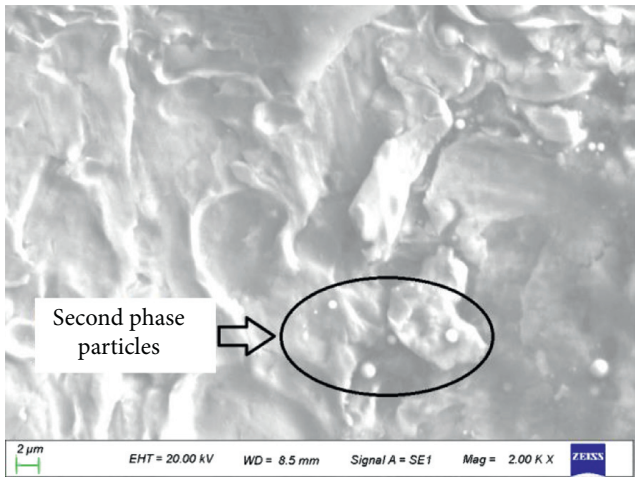

(d)

Figure 6: Macro- and micromorphology of fatigue fracture. (a) The macroscopic morphology of the fatigue fracture. (b) The fatigue crack propagation region (1000X). (c) The final fracture region (1000X). (d) The final fracture region (2000X).

process. The larger the $\Delta \alpha$ value, the wider the range of height fluctuation. At this time, the surface of the specimen fluctuates violently, and the multifractal spectrum becomes wider. Conversely, the smaller the $\Delta \alpha$ value, the smaller the height fluctuation range. The specimen surface is flat, and the multifractal spectrum becomes narrower. 


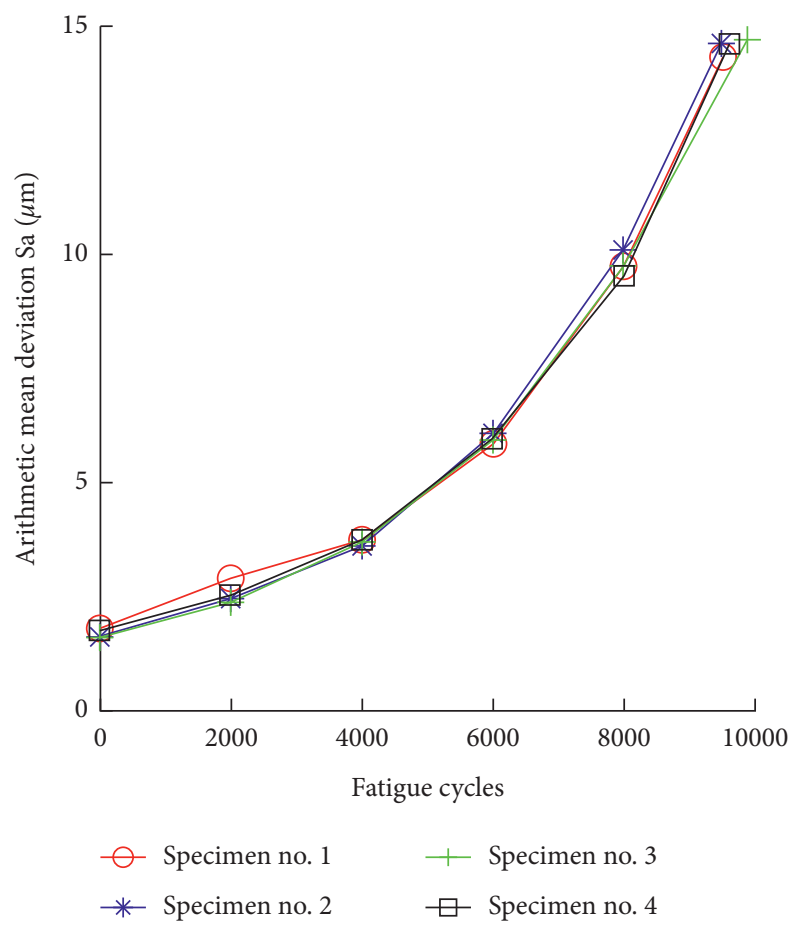

FIgURE 7: Changes of $S_{a}$ in the fatigue process.

Figure 9 shows the multifractal spectrum width variation curve of four specimens in the fatigue process. It is clear that $\Delta \alpha$ increases with the increase of fatigue cycles, indicating that the height fluctuation range of surface topography increases with the increment of fatigue cycles, and the surface changes from smooth to rough.

4.4. Height Frequency Distribution and Digital Characteristic Analysis. The metal surface height data collected for each damage state are divided into intervals of $5 \mu \mathrm{m}$. For example, when the fatigue cycle of no. 1 specimen is 6000 , the maximum and minimum surface height data are $15.09 \mu \mathrm{m}$ and $-27.2 \mu \mathrm{m}$, respectively. Therefore, the surface height is divided into the following 10 intervals: $(-30,-25),(-25$, $-20),(-20,-15),(-15,-10),(-10,-5),(-5,0),(0,5),(5,10)$, $(10,15)$, and $(15,20)$, and calculate the number of height data in accordance with the abovementioned intervals. The frequency distribution curve of metal surface height is shown in Figure 10. With the increase of fatigue cycles, the concentration of metal surface height data decreases, and the span becomes larger.

The normality of the surface height distribution of the four specimens during the fatigue process is tested, and it is found that the surface height distribution of each damage state obeys the normal distribution. Figure 11 shows the normality test results of the surface height distribution of no. 1 specimen in each damage state.

Figures 12-14 are the variation curves of the numerical characteristics (amplitude A, mean $\mu$, and standard deviation $\delta$ ) of the surface height distribution of the four specimens during fatigue. The amplitude A decreases with the increase of fatigue cycles, while the mean $\mu$ and standard deviation $\delta$ increase with the increase of fatigue cycles, indicating that the concentration of metal surface height data decreases during the fatigue process, and the proportion of the main height value is increasing, the surface becomes uneven, and the roughness increases.

\section{Topography Feature Data Fusion and Fatigue Damage Assessment Based on Bayesian Estimation}

5.1. Prior Probability Analysis. Through the low-cycle fatigue test, 24 sets of data with 6 damage states $(0,2000,4000,6000$, 8000 cycles, and fracture) were obtained, of which 18 sets of data were as training data and 6 sets of data were as test data. Taking 0 cycle as an example, the mean and variance (i.e., prior data) of its characteristic parameters were calculated, as shown in Table 1. The prior data of each damage state can also be obtained. Figure 15 shows the probability density diagram of each characteristic parameter under different fatigue damage states.

5.2. Posterior Probability Analysis. Table 2 shows the 6 sets of test data for each fatigue damage state, and the posterior probability values of the test data were obtained based on the prior probability from the training data (Table 3 ).

From the posterior probability of the test data in Table 3, a series of posterior probability values were obtained for each group of test data, and the prediction loading cycles corresponding to the maximum value were taken as the prediction result. It is found that the predicted results matched the actual categories, and the Naive Bayesian method could be used for the data fusion of surface topography features in the fatigue damage process.

\section{Discussion}

By analyzing the data of 3D surface topography of the Q235 steel specimen during the fatigue damage process, it is found that the statistical characteristics of the above 3D topography, such as arithmetic mean deviation $S_{a}$, surface height distribution mean $\mu$, and standard deviation $\delta$. show obvious regular changes with the increase of fatigue cycles. Although the experiment was carried out on a Q235 material, the feature extraction method and corresponding rules can also be applied to other carbon steel materials, such as 45 steel and Q345.

In the process of low-cycle fatigue damage, significant plastic deformation will be generated during each cycle of loading, and its fatigue behavior can be related to plastic deformation and limited fatigue cycles. The fatigue damage process consists of three stages: microstructure evolution, crack initiation, and propagation, and the crack initiation life accounts for the majority of the total life. The low-cycle fatigue cracks preferentially nucleate in the persistent slip band (PSB) with the highest slip inhomogeneity and maximum strain localization. The slip produced by the PSB in the surface grains can be transferred in a large amount, and the phenomenon of 

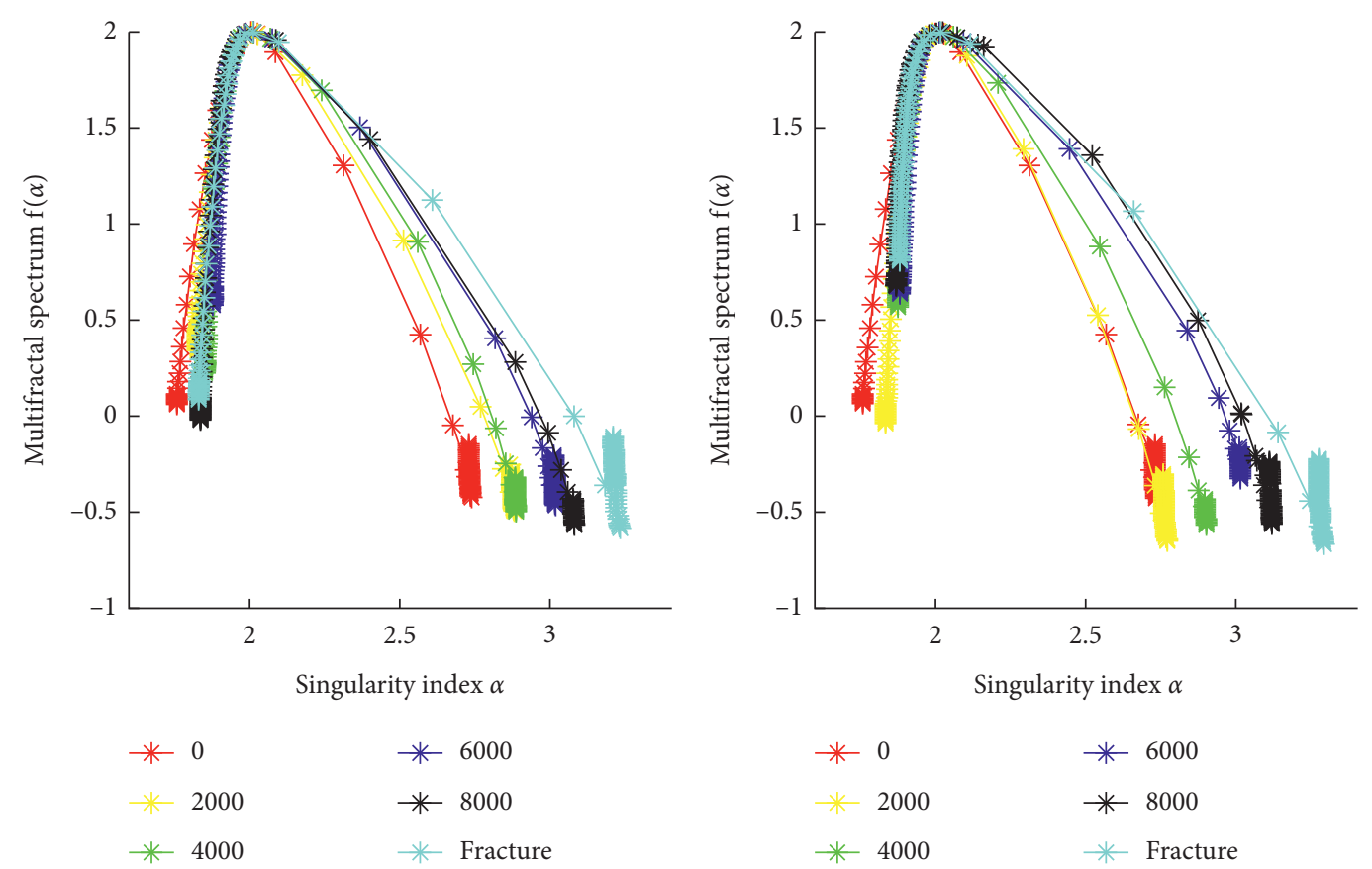

(a)
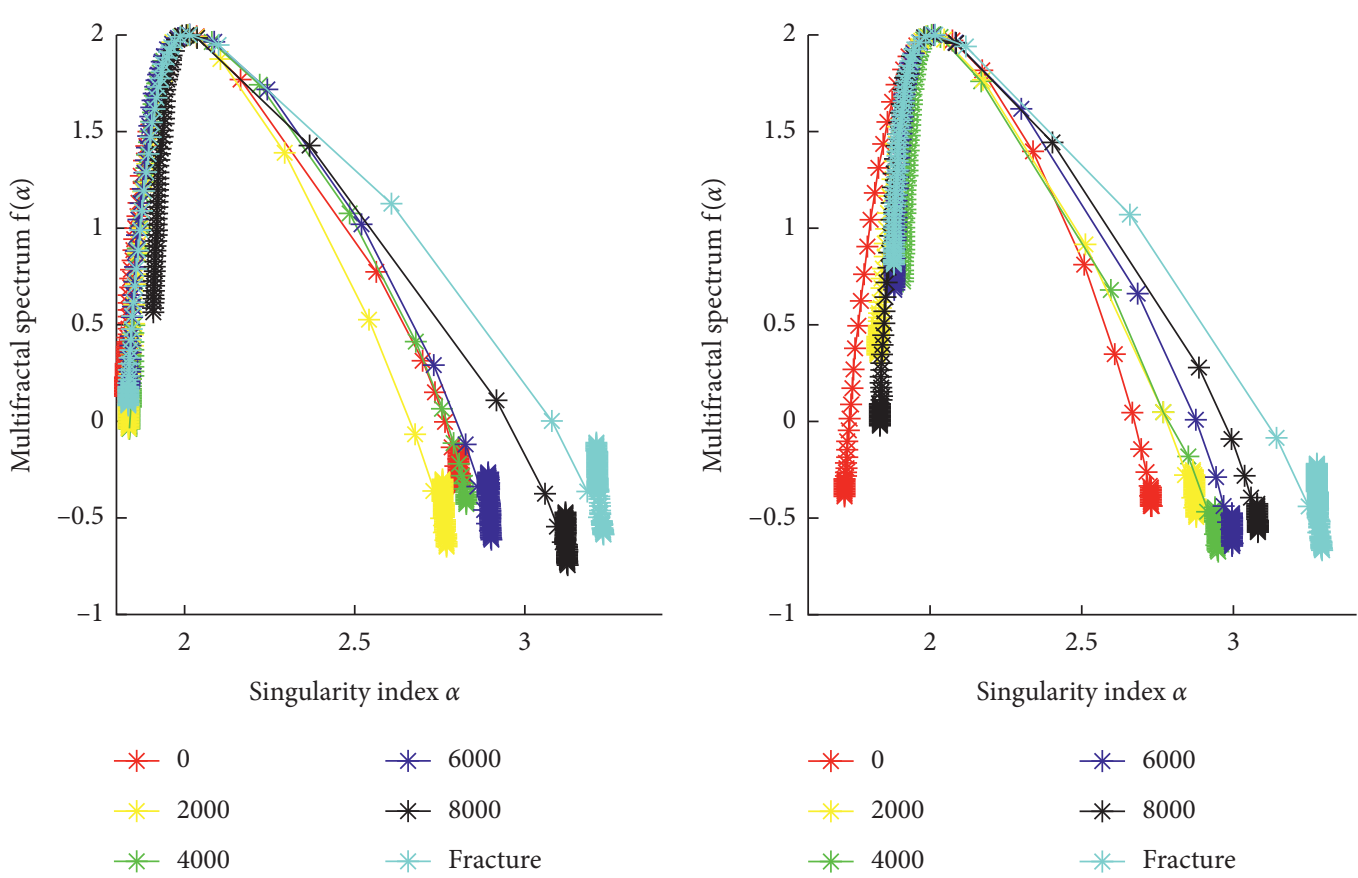

(c)

(d)

FIGURE 8: The surface multifractal spectrum of the metal fatigue process. (a) Specimen no. 1. (b) Specimen no. 2. (c) Specimen no. 3. (d) Specimen no. 4.

material intrusion and extrusion caused by this slip band can be observed on the free surface of the specimen. So far, the mechanism of crack initiation caused by this intrusion and extrusion is still unclear, which may be related to the dynamic equilibrium between dislocation proliferation and annihilation in the PSB. The whole process of crack initiation, propagation, and fracture was described by fatigue fracture analysis, such as the nucleation of microcracks at the specimen surface, fatigue striations caused by crack propagation, dimple morphology, and second phase particles in the transient fracture region. The nature of fracture is verified, and the necessary means are provided to reveal the fracture mechanism.

This paper presents the statistical results of surface topography features and describes the quantitative 


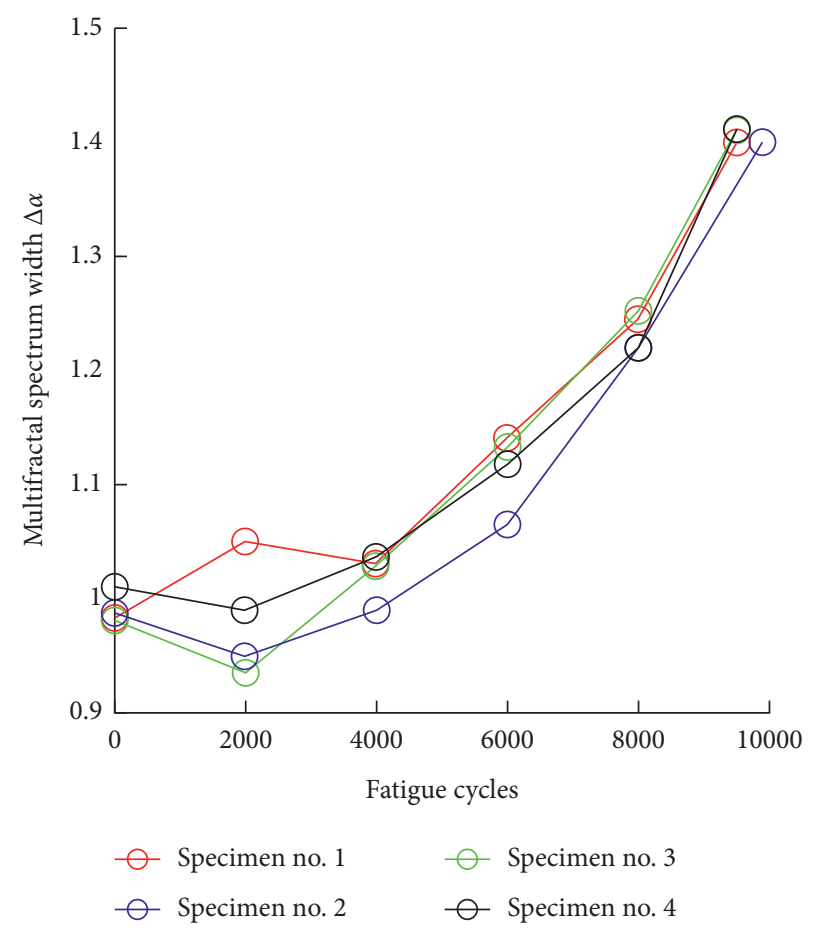

FIgURE 9: Variation curves of multifractal spectrum width.

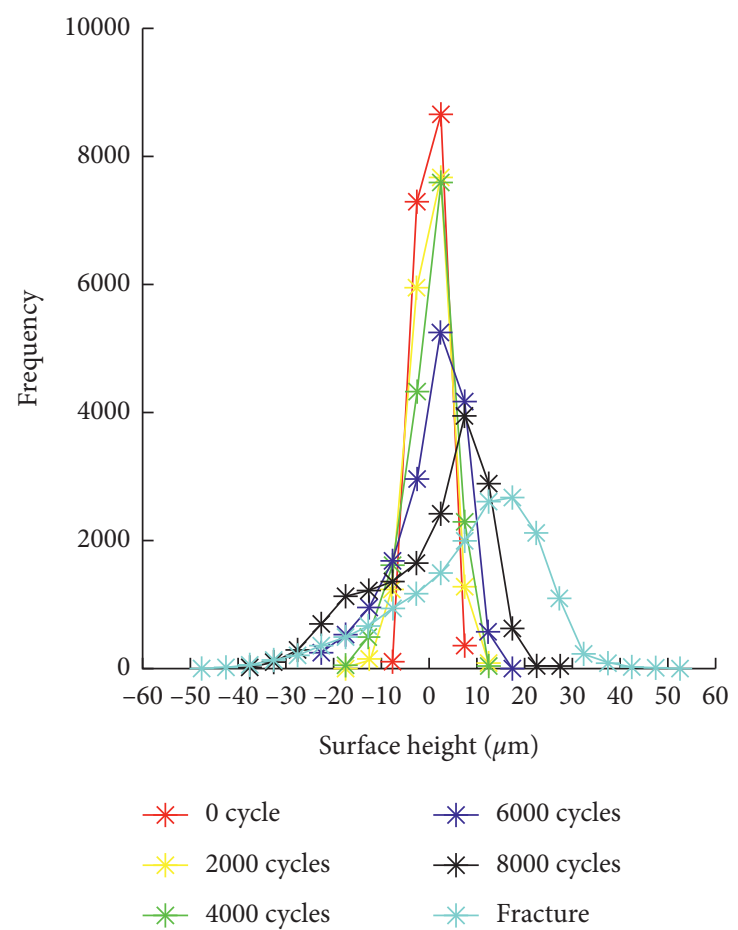

(a)

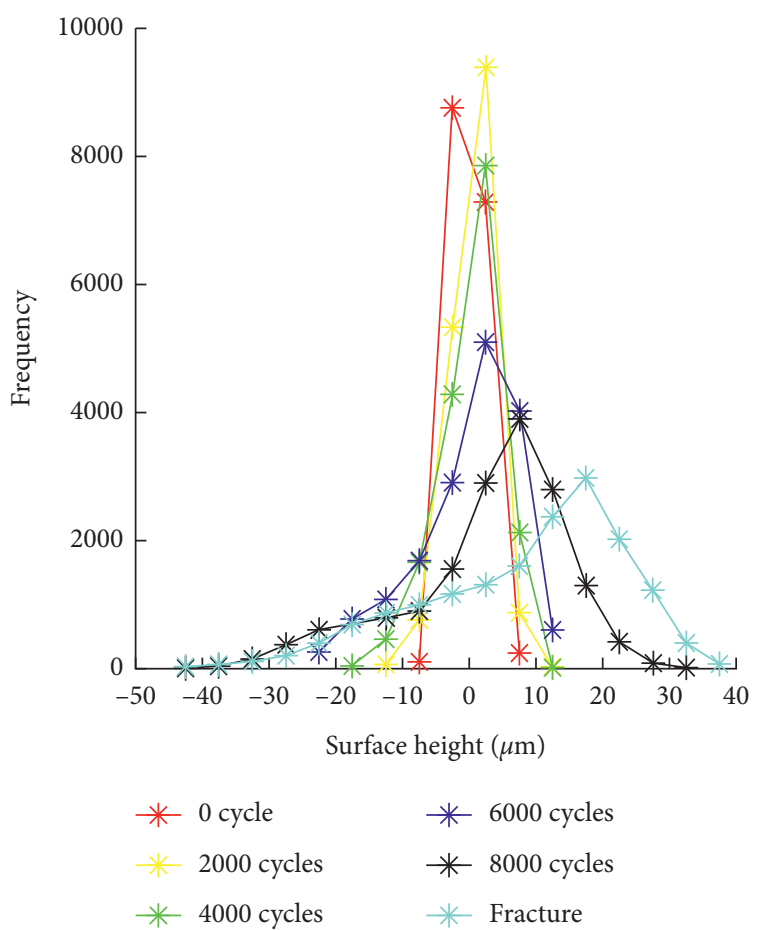

(b)

Figure 10: Continued. 


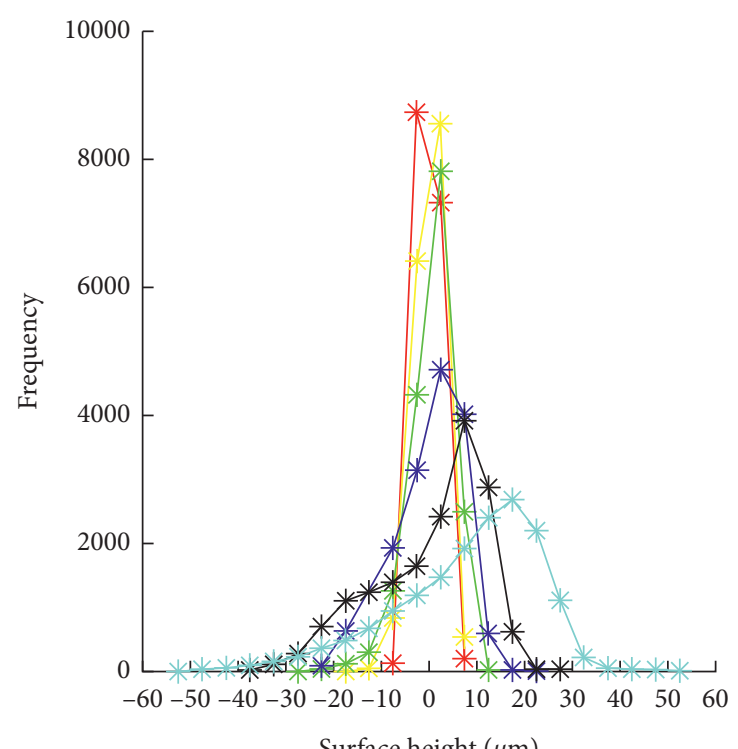

Surface height $(\mu \mathrm{m})$

$$
\begin{array}{ll}
* 0 \text { cycle } & * 6000 \text { cycles } \\
* 2000 \text { cycles } & * 8000 \text { cycles } \\
* 4000 \text { cycles } & * \text { Fracture }
\end{array}
$$

(c)

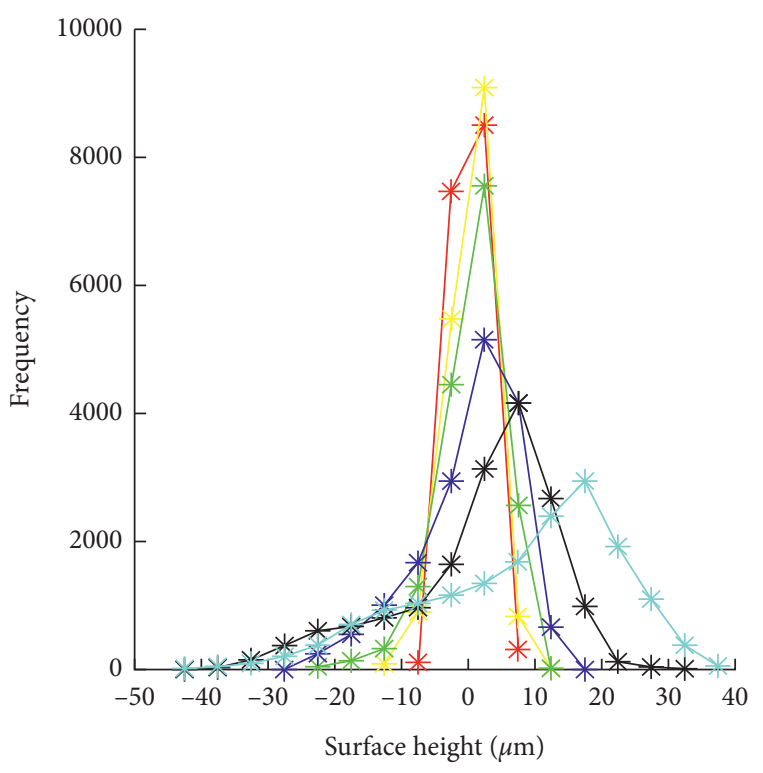

$$
\begin{array}{ll}
* 0 \text { cycle } & * 6000 \text { cycles } \\
* 2000 \text { cycles } & * 8000 \text { cycles } \\
* 4000 \text { cycles } & * \text { Fracture }
\end{array}
$$

(d)

FIgURE 10: The surface height frequency distribution curve of the metal fatigue process. (a) Specimen no. 1. (b) Specimen no. 2. (c) Specimen no. 3. (d) Specimen no. 4.

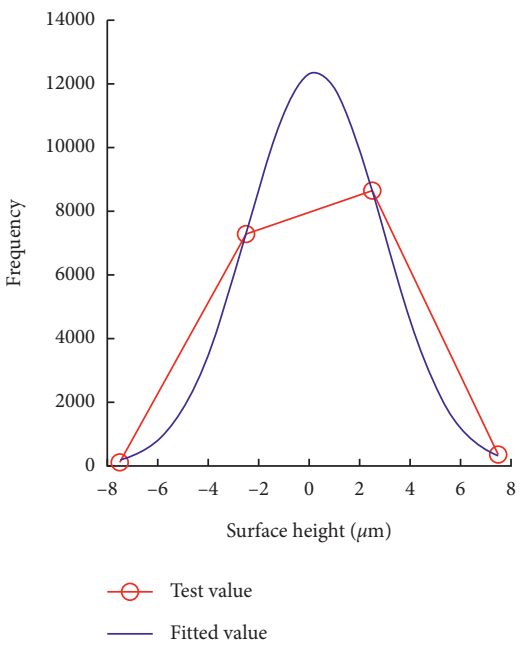

(a)

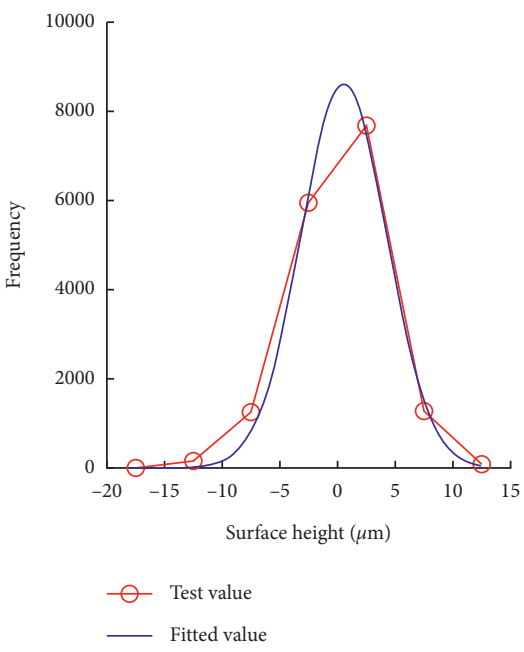

(b)

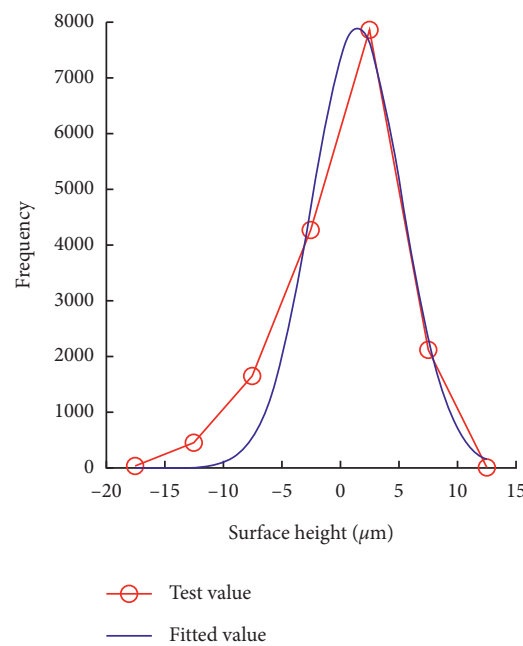

(c)

Figure 11: Continued. 


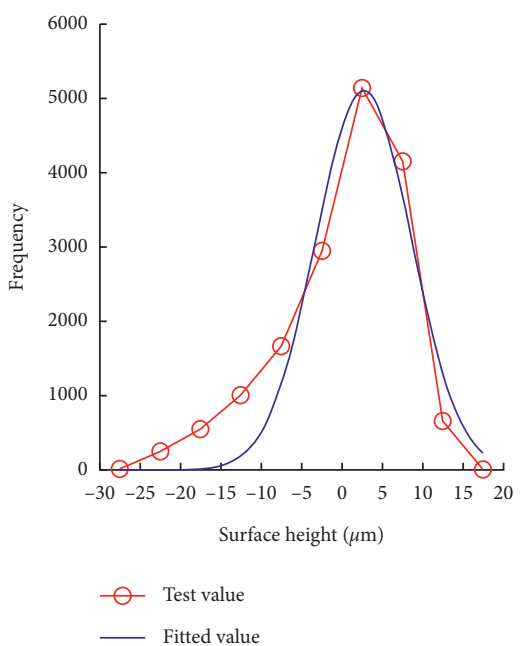

(d)

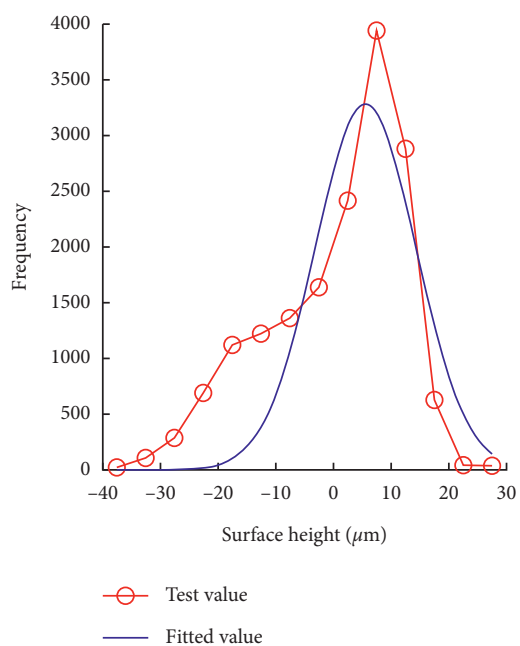

(e)

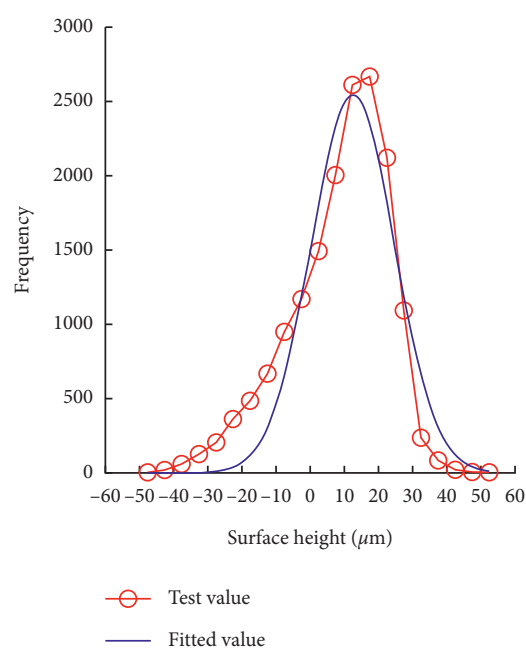

(f)

Figure 11: The normality test of surface height distribution during fatigue. (a) 0 cycle. (b) 2000 cycles. (c) 4000 cycles. (d) 6000 cycles. (e) 8000 cycles. (f) Fracture.

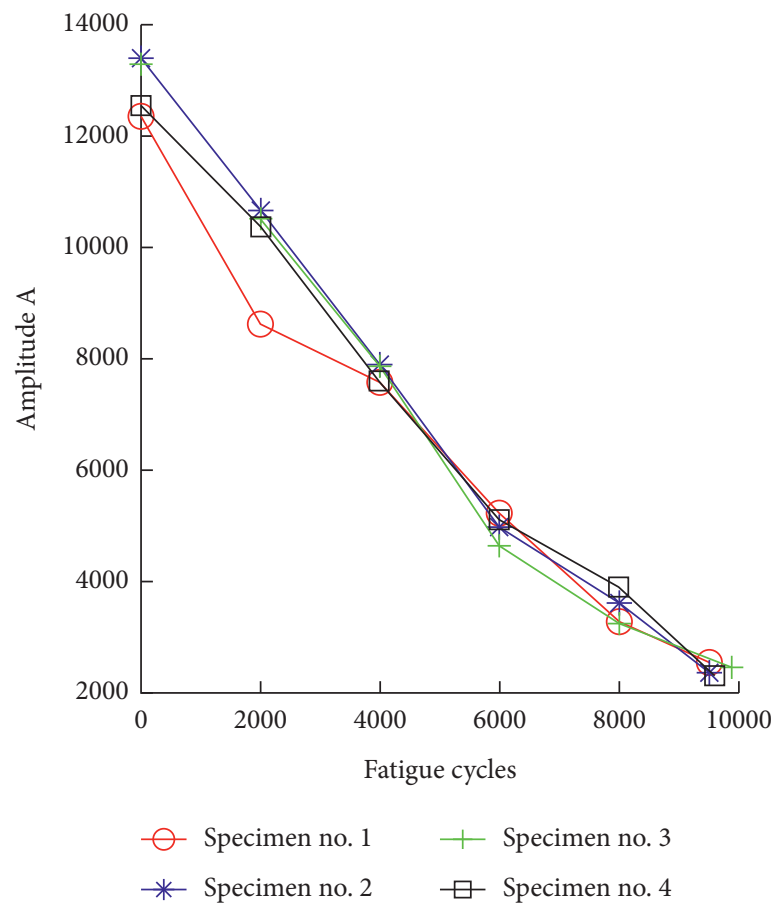

Figure 12: The variation curves of amplitude (A).

relationship between them and fatigue cycles, aiming to establish a data fusion assessment model for fatigue damage by constructing a nonlinear mapping between surface topography features and damage. In this way, a feasible operational method for fatigue damage assessment in engineering is sought to avoid the current situation where the micromechanism (such as crack nucleation, vacancy dipole, and interface dislocation.) of the fatigue damage process is 


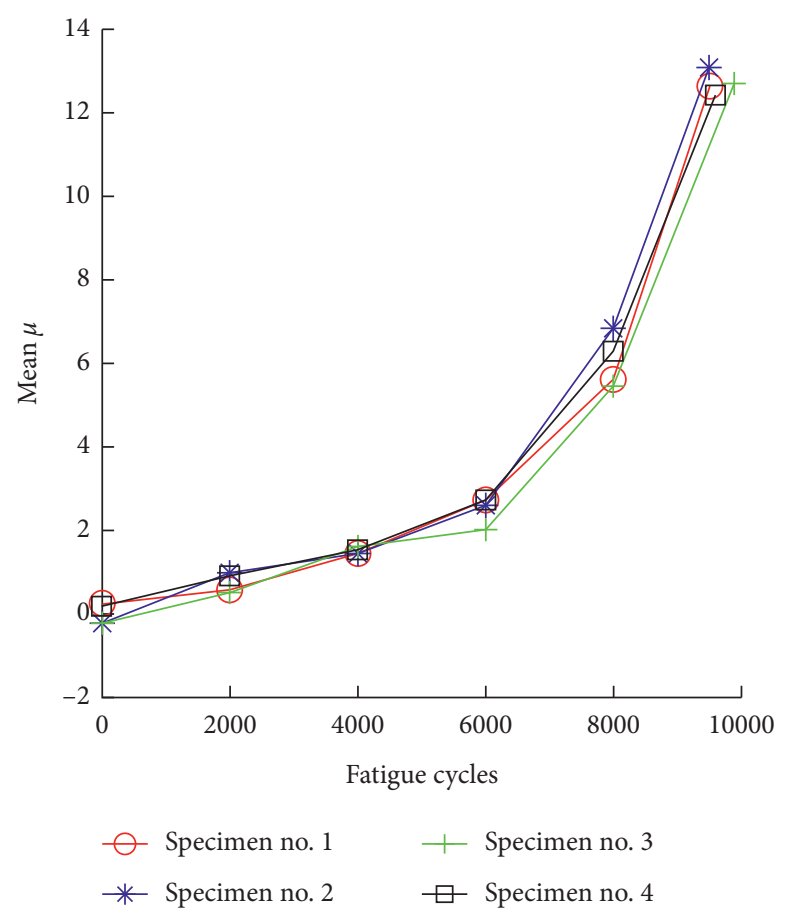

FIGURE 13: The variation curves of mean value $\mu$.

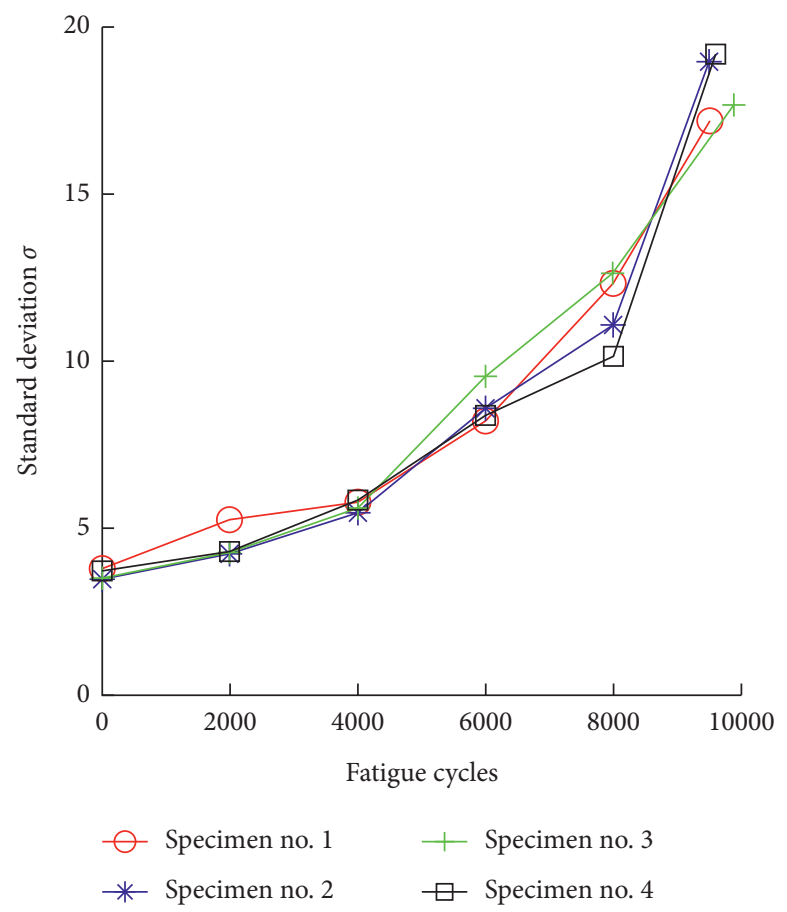

Figure 14: The variation curves of standard deviation $\delta$.

TABLE 1: Mean and variance values of each characteristic parameter $(0$ cycle).

\begin{tabular}{lcc}
\hline Characteristic parameter & Mean & Variance \\
\hline$\mu$ & 0.2294 & $1.57 \times 10^{-4}$ \\
$\delta$ & 3.5936 & $1.67 \times 10^{-2}$ \\
$A$ & 13013 & $2.22 \times 10^{5}$ \\
$S_{a}$ & 1.6789 & $1.02 \times 10^{-2}$ \\
$\Delta \alpha$ & 0.9839 & $6.32 \times 10^{-6}$ \\
\hline
\end{tabular}




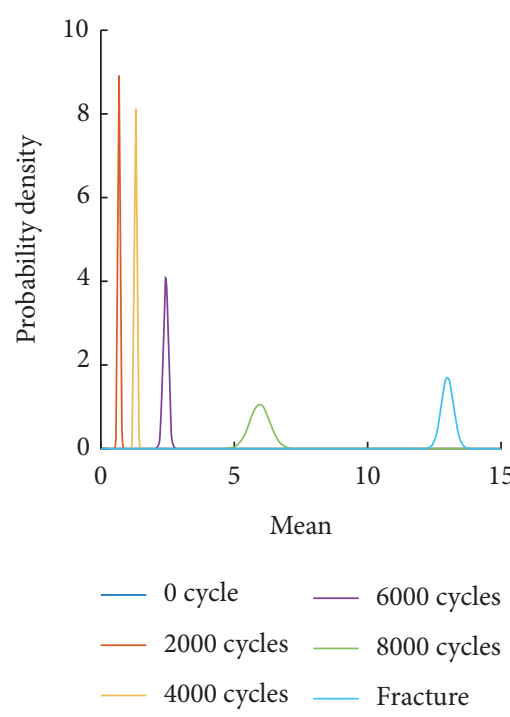

(a)

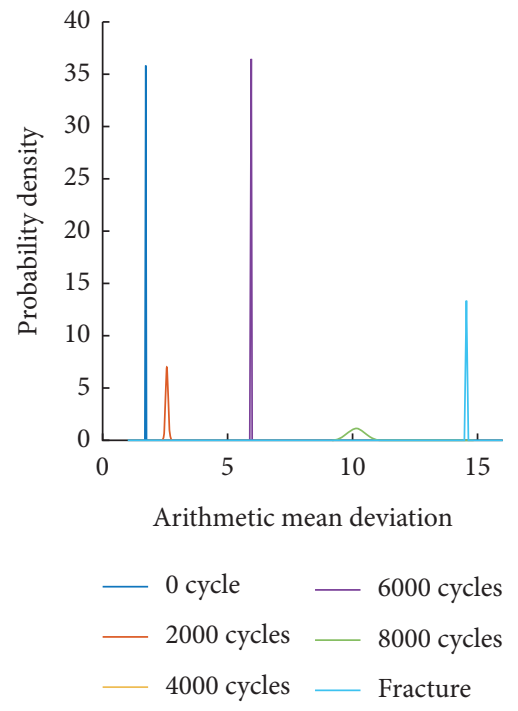

(d)
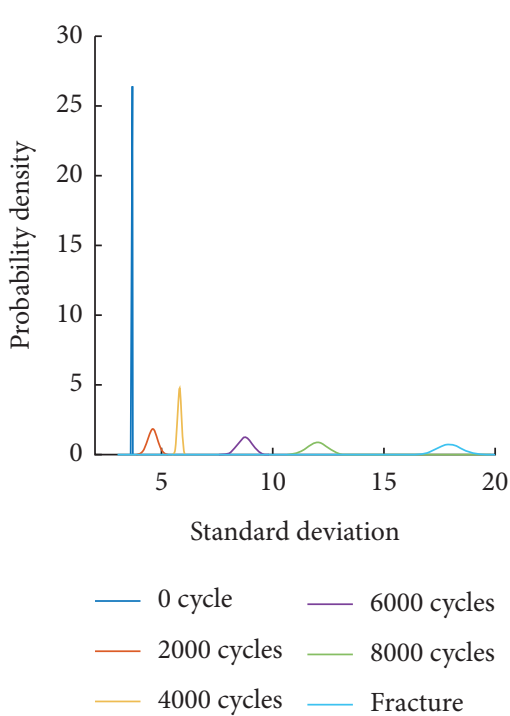

(b)

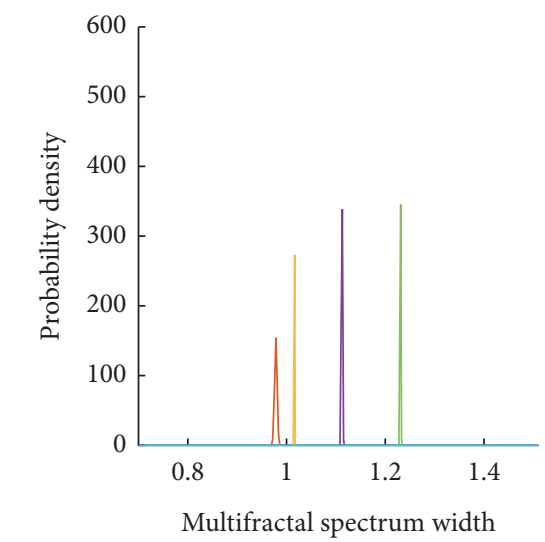

(e)

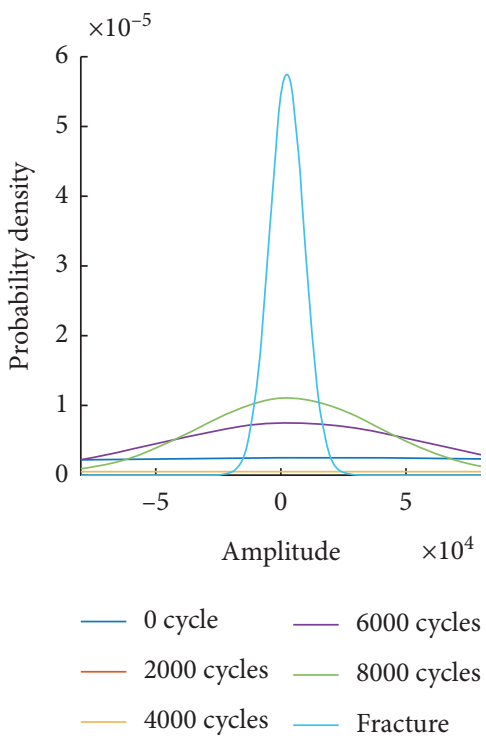

(c)

FIGURE 15: The priori probability distribution of each characteristic parameter under different cycles. (a) $\mu$. (b) $\delta$. (c) $A$. (d) $S_{a \text {. }}$ (e) $\Delta \alpha$.

TABLe 2: Six sets of test data values for each damage state.

\begin{tabular}{lccccc}
\hline Fatigue cycle & $\mu$ & $\delta$ & $A$ & $S_{a}$ & \multicolumn{1}{c}{0} \\
\hline 0 & 0.224 & 3.487 & 13400 & 1.6152 & 2.0777 \\
2000 & 0.9022 & 4.309 & 10370 & 3.7357 & 0.9913 \\
4000 & 1.531 & 5.835 & 7598 & 5.9485 & 1.0365 \\
6000 & 2.72 & 8.375 & 5104 & 9.5093 & 1.1175 \\
8000 & 6.285 & 10.15 & 3898 & 1.22 \\
Fracture & 12.42 & 19.1937 & 2314 & 1.4985 \\
\hline
\end{tabular}


TABLe 3: Posterior probability values of test data.

\begin{tabular}{|c|c|c|c|c|c|c|}
\hline \multicolumn{7}{|c|}{ Test data } \\
\hline Predicted loading cycles & 0 cycle & 2000 cycles & 4000 cycles & 6000 cycles & 8000 cycles & Facture \\
\hline 0 & $2.146 \times 10^{-2}$ & $8.75 \times 10^{-161}$ & $2.624 \times 10^{-749}$ & $8.717 \times 10^{-1395}$ & $9.498 \times 10^{-4302}$ & $\approx 0$ \\
\hline 2000 & $1.779 \times 10^{-12}$ & $6.915 \times 10^{-5}$ & $1.425 \times 10^{-15}$ & $1.0 \times 10^{-91}$ & $1.96 \times 10^{-391}$ & $9.313 \times 10^{-1490}$ \\
\hline 4000 & $2.06 \times 10^{-362}$ & $6.656 \times 10^{-212}$ & $4.947 \times 10^{-3}$ & $4.33 \times 10^{-431}$ & $4.436 \times 10^{-2808}$ & $\approx 0$ \\
\hline 6000 & $2.13 \times 10^{-713}$ & $4.3 \times 10^{-455}$ & $9.0 \times 10^{-142}$ & $4.776 \times 10^{-3}$ & $8.35 \times 10^{-308}$ & $4.799 \times 10^{-1901}$ \\
\hline 8000 & $3.9 \times 10^{-727}$ & $2.312 \times 10^{-400}$ & $1.558 \times 10^{-178}$ & $2.15 \times 10^{-49}$ & $2.59 \times 10^{-7}$ & $1.196 \times 10^{-81}$ \\
\hline Fracture & $\approx 0$ & $3.57 \times 10^{-3459}$ & $1.19 \times 10^{-3231}$ & $2.83 \times 10^{-1743}$ & $2.877 \times 10^{-655}$ & $5.78 \times 10^{-4}$ \\
\hline
\end{tabular}

unclear and difficult to quantify the damage. It is becoming a hot issue to correlate the microscale deformation mechanism of metal materials/components with fatigue failure at the macroscale.

\section{Conclusion}

Q235 steel specimens were selected for tensile fatigue tests, and the three-dimensional surface topography information was extracted. The characteristics of metal surface topography during the fatigue process, such as arithmetic mean deviation $S_{a}$, surface height distribution amplitude $A$, mean $\mu$, standard deviation $\delta$, and multifractal spectrum width $\Delta \alpha$, were obtained.

It is found that $S_{a}$ shows a nonlinear growth trend with the increase of fatigue cycles. Due to the initiation and expansion of cracks in the middle and late stages of fatigue, the prominent peaks and valleys are higher and deeper, and the growth rate of $S_{a}$ becomes faster. In the digital features of surface height distribution, the amplitude $A$ decreases with the increase of fatigue cycles, and the mean value $\mu$ and standard deviation $\delta$ increase with the increase of fatigue cycles. The multifractal spectrum width $\Delta \alpha$ increases with the increment of fatigue cycles, indicating that the fluctuation range of metal surface height data increases with the increase of fatigue damage, and the surface becomes uneven.

The arithmetic mean deviation $S_{a}$, digital features of surface height distribution (amplitude $A$, mean $\mu$, and standard deviation $\delta$ ), and multifractal spectral width $\Delta \alpha$ were taken as the multifeature quantities of surface topography. The data fusion of the above multifeature quantities was performed based on the Naive Bayesian algorithm, and its validity was preliminarily verified.

This study can provide methodological support for fatigue damage assessment of mechanical components. However, the amount of experimental data in the modeling process is still small, which needs to be increased. In addition, in order to improve the prediction accuracy and reduce the uncertainty, the subsequent research will conduct fusion assessment based on topographic features and combined with multiple physical parameters, which is also an important direction for future nondestructive testing.

\section{Data Availability}

The data used to support the findings of this study are available from the corresponding author upon request.

\section{Conflicts of Interest}

The authors declare that there are no conflicts of interest regarding the publication of this paper.

\section{Acknowledgments}

This work was supported by the Natural Science Foundation of China (Grant no. 51805003), Anhui Education Department Excellent Young Talent Support Project (Grant no. gxyqZD2019057), Natural Science Research Project of Anhui Education Department (Grant no. KJ2018A0519), and Natural Science Foundation of Anhui Province (Grant nos. 1808085ME125 and 1908085QE243).

\section{References}

[1] S. Ma and H. Yuan, "A continuum damage model for multiaxial low cycle fatigue of porous sintered metals based on the critical plane concept," Mechanics of Materials, vol. 104, pp. 13-25, 2017.

[2] X. Y. Tong, H. X. Li, L. J. Yao, and B. Li, "Feature extraction and analysis of surface microscopic image of pure copper subjecting low cycle fatigue," Mechanical Science and Technology for Aerospace Engineering, vol. 34, no. 9, pp. 1446-1450, 2015.

[3] D. Zhu, L. Xu, F. Wang, T. Liu, and K. Lu, "Evolution of metal surface topography during fatigue," Metals, vol. 7, no. 2, Article ID 66, 2017.

[4] N. Fujimura, T. Nakamura, H. Oguma, T. Nakajima, and T. Shinko, "Fatigue damage assessment of SUS316NG based on surface roughness measurement and the applicable scope," Journal of the Society of Materials Science, Japan, vol. 62, no. 12, pp. 750-755, 2013.

[5] A. B. Pradana and P. Prajitno, "A Portable Surface Roughness Measurement System Using Laser Speckle Imaging Based on GLCM," in 2019 6th International Conference On Instrumentation, Control, and Automation (ICA), pp. 100-105, IEEE, Bandung, Indonesia, August 2019.

[6] N. Fujimura, T. Nakamura, and H. Oguma, "Changes in surface roughness during low cycle fatigue process of austenitic stainless steel," Journal of Solid Mechanics and Materials Engineering, vol. 7, no. 3, pp. 372-380, 2013.

[7] Y. Wang, E. I. Meletis, and H. Huang, "Quantitative study of surface roughness evolution during low-cycle fatigue of $316 \mathrm{~L}$ stainless steel using Scanning Whitelight Interferometric (SWLI) Microscopy," International Journal of Fatigue, vol. 48, pp. 280-288, 2013.

[8] A. Haghshenas and M. M. Khonsari, "Damage accumulation and crack initiation detection based on the evolution of surface roughness parameters," International Journal of Fatigue, vol. 107, pp. 130-144, 2018.

[9] J. F. Sola, R. Kelton, E. I. Meletis, and H. Y. Huang, "A surface roughness based damage index for predicting future propagation path of microstructure-sensitive crack in pure nickel," International Journal of Fatigue, vol. 122, pp. 164-172, 2019. 
[10] A. Kato and F. Okuya, "Evaluation of fatigue damage using laser speckle: relation between surface profile and diffraction pattern," Proceedings of SPIE - The International Society for Optical Engineering, vol. 4317, pp. 374-379, 2001.

[11] P. Zuluaga-Ramírez, M. Frövel, T. Belenguer, and F. Salazar, "Non contact inspection of the fatigue damage state of carbon fiber reinforced polymer by optical surface roughness measurements," NDT \& E International, vol. 70, pp. 22-28, 2015.

[12] J. F. Sola, R. Kelton, E. I. Meletis, and H. Y. Huang, "Predicting crack initiation site in polycrystalline nickel through surface topography changes," International Journal of Fatigue, vol. 124, pp. 70-81, 2019.

[13] X. Sun and Z. Q. Wu, "Fractal and multifractal description of surface topography," Acta Physica Sinica, vol. 50, no. 11, pp. 2126-2131, 2001.

[14] B. C. Wang and J. H. Zhu, "Statistical characteristics of electrochemically polished surface topography of mild steel," Journal of Xi'an Jiaotong University, vol. 05, pp. 106-110, 2006.

[15] B. F. He, C. E. Wei, and B. X. Liu, "Three-dimensional surface roughness characterization and application," Optics and Precision Engineering, vol. 26, no. 08, pp. 164-181, 2018.

[16] Geometrical Product Specifications, (GPS)-Surface Texture: Areal-Part 604: Nominal Characteristics of Non-contact (Coherence Scanning Interferometry) Instruments, ISO, Geneva, Switzerland, 2013. 